\title{
Destinos Turísticos Inteligentes: Una perspectiva desde la inclusión social y la participación comunitaria
}

\author{
Salessi María Lucila \\ Universidad Nacional de La Plata, Facultad de Ciencias Económicas Argentina. \\ Institituto de Investigaciones en Turismo. Argentina \\ Calle 6 777, La Plata, Buenos Aires, B1900TEN La Plata, Buenos Aires. \\ lucila.salessi@econo.unlp.edu.ar
}

\section{Resumen}

Pensar los destinos turísticos inteligentes como espacios comunes, interconectados, de convivencia, respeto y enriquecimiento mutuo entre turistas y residentes, requiere, sin dudas, de un cambio de paradigma. El desafío es hacia la co-gestión entre la pluralidad de actores que conforman/integran el sector turístico y la toma de decisiones consensuada y legitimada por los mismos, en un escenario en el cual la tecnología, la innovación, la sostenibilidad y la accesibilidad generen nuevas experiencias y modelos inclusivos para repensar el turismo.

El presente trabajo parte de la premisa de que la inclusión y la participación social constituyen elementos intrínsecos a los espacios y destinos turísticos, para que de esa forma inteligencia e inclusión se vinculen entre sí, trasladando a los mismos el derecho que tienen las personas de legitimar sus prácticas de inmersión social en el marco del desarrollo de la actividad turística.

\begin{abstract}
Thinking smart tourist destinations as common spaces, interconnected, of coexistence, respect and mutual enrichment between tourists and residents, requires definitely a paradigm shift. The challenge is towards co-management between the plurality of actors that make up/integrate the tourism sector and the decision-making process agreed and legitimized by them, in a scenario in which technology, innovation, sustainability and accessibility generate new experiences and inclusive models to rethink tourism.
\end{abstract}


Actas del Seminario Internacional Destinos Turísticos Inteligentes:

nuevos horizontes en la investigación y gestión del turismo

Universidad de Alicante, 26 y 27 de octubre de 2017

The present work is based on the premise that inclusion and social participation are intrinsic elements to tourist spaces and destinations, so that intelligence and inclusion are linked to each other, transferring to them the right that people have to legitimize their practices of social immersion in the framework of the development of the tourist activity.

Keywords: inclusión social, destinos turísticos inteligentes, participación comunitaria, turismo.

\section{Introducción}

El marco del Año Internacional del Turismo Sostenible para el Desarrollo 2017 constituye un incentivo para fomentar el diálogo social y político que promueva iniciativas, inversiones y acciones gubernamentales conducentes al desarrollo y a la lucha contra la pobreza. En este sentido, creemos que los destinos turísticos inteligentes deberán fomentar dicho diálogo social, entre la totalidad de actores que conforman el sistema turístico abogando por la construcción de espacios urbanos de convivencia y respeto entre turistas y residentes.

Inclusión e innovación en el marco de un destino turístico inteligente (DTI) significa que la actividad turística se instale en las agendas de gobierno, como un verdadero adalid del desarrollo sostenible, generando empleos genuinos, creando instancias de capacitación y formación profesional, mejorando la infraestructura y la conectividad de las ciudades, haciéndola más accesible para todos, compartiendo experiencias comunitarias y colaborativas.

El turismo como un fenómeno económico y social, se sostiene en el desarrollo local y su contribución a la cohesión social. Así, los destinos turísticos inteligentes DTI deberían generar oportunidades concretas de integración e inclusión, creando legítimas fuentes de trabajo vinculadas a toda la cadena de valor y prestación de productos y servicios turísticos.

Pensar los destinos turísticos como espacios comunes interconectados de convivencia, respeto y enriquecimiento mutuo entre turistas y residentes, y que su vez sean grandes generadores de experiencias, requiere de un proceso de cambio de paradigma hacia un modelo en el cual se generen planes, programas y proyectos abiertos, flexibles y participativos. La apuesta es hacia la co-gestión entre la pluralidad de actores que conforman/integran el sector turístico (Domínguez y Talavera, 2016), la toma de decisiones 
Actas del Seminario Internacional Destinos Turísticos Inteligentes:

nuevos horizontes en la investigación y gestión del turismo

Universidad de Alicante, 26 y 27 de octubre de 2017

consensuada y legitimada por los mismos en un escenario en el cual la tecnología, la innovación, la sostenibilidad y la accesibilidad generen nuevos modelos inclusivos para repensar el turismo.

El presente trabajo parte de la premisa de que la inclusión y la participación social constituye un elemento que necesita ser aplicado a los espacios y destinos turísticos, para que de esa forma inteligencia e inclusión se vinculen entre sí, trasladando a los mismos el derecho que tienen las personas de legitimar sus prácticas de inmersión social en el marco del desarrollo de la actividad turística.

Dentro de la conceptualización de Destinos Turísticos Inteligentes, nos interesa especialmente destacar y resaltar la dimensión social que se observa en cuanto a las prestaciones vinculadas a servicios turísticos y no turísticos que hacen a la mejora de la calidad de vida de los residentes.

Usar la tecnología al servicio del ser humano, en el sector turístico se traduce en potenciar al máximo la relación experiencial que se genera entre turistas y residentes, un vínculo en el que la creatividad y la legitimidad del encuentro se pone por encima de cualquier otra práctica convencional de prestación de servicios turísticos.

Ahora bien, en el marco de la construcción y gestión de un destino turístico inteligente, entendemos que uno de los retos más importantes de la gestión y administración pública es la creación de comunidades ricas en información, que promuevan la protección social interconectada, capaz de generar por sí misma un aprendizaje continuo sobre cómo pueden aumentar las oportunidades de sus residentes en cuanto a la prestación de productos y servicios turísticos.

Una ciudad o urbe inteligente debe ser pensada como un sistema orgánico - una red de interrelaciones, un sistema interconectado, el cual se sostiene a través de su permanente activación y fomento por su administración y gobierno público en convivencia con sus ciudadanos. El modelo de gestión se basa en la propuesta de valor agregado puesta en la relación entre gobierno, sociedad y gobernanza local solidaria, siendo sus principales premisas la pluralidad democrática y la responsabilidad. (Macadar et al 2013)

En esta misma línea de análisis, podemos entender la inclusión social como la inteligencia urbana al servicio del ciudadano. Una ciudad más justa y participativa promoverá espacios ciudadanos de diálogo, acuerdos, negociaciones y consenso que puedan dirimir los conflictos y superar las 
Actas del Seminario Internacional Destinos Turísticos Inteligentes:

nuevos horizontes en la investigación y gestión del turismo

Universidad de Alicante, 26 y 27 de octubre de 2017

desigualdades, facilitando herramientas de integración y de justicia social entre sus habitantes.

\section{Metodología}

El presente trabajo de carácter exploratorio, se propone realizar una revisión bibliográfica y análisis documental que contribuya a analizar un estado de la cuestión respecto de las prácticas de inclusión social y su imbricación en los denominados destinos turísticos inteligentes.

Dada la complejidad y multiplicidad de perspectivas y enfoques para la construcción de un marco conceptual que dé cuenta de la conceptualización de los destinos turísticos inteligentes, resulta indispensable realizar una revisión de la literatura que reúna definiciones de ciudades inteligentes, gestión de destinos turísticos, territorios inteligentes, etc.

Respecto del análisis bibliográfico: en primera instancia se recurrirá al relevamiento de documentos sobre la temática y posterior análisis de contenido (Sautu et al, 2005), a fin de lograr definir conceptos básicos consecuentes con el desarrollo del trabajo, como por ejemplo el concepto de destino turístico inteligente, inclusión social, participación comunitaria. Asimismo, el método seleccionado para comprender e identificar cada una de las prácticas participativas de inclusión social en el contexto de los llamados destinos turísticos inteligentes, será el análisis documental e histórico y el estudio bibliográfico de diversos tipos de fuentes académicas, institucionales, periodísticas, documentales, entre otros.

\section{Destinos Turísticos Inteligentes. Una aproximación teórica y conceptual}

Un destino turístico innovador que garantiza el desarrollo sostenible e inclusivo de su territorio, que se sostiene en un soporte tecnológico de vanguardia, es accesible y facilita la interacción del viajero con su entorno en un ambiente limpio y sostenible potenciando e incrementando la calidad de la experiencia y encuentro turístico; se puede entender y conceptualizar como un Destino Turístico Inteligente.

Los aspectos positivos que permiten sostener un proceso de reestructuración y reconversión hacia un DTI radican principalmente en la mejora de la competitividad del destino basada fundamentalmente en su capacidad de gestión e innovación. Estas acciones y líneas estratégicas permitirán 
Actas del Seminario Internacional Destinos Turísticos Inteligentes:

nuevos horizontes en la investigación y gestión del turismo

Universidad de Alicante, 26 y 27 de octubre de 2017

acercar, disponer y facilitar el uso y apropiación tecnología de su patrimonio, recursos naturales y culturales consecuentes con una adecuada y eficiente comunicación y percepción de lo que el destino puede y tiene para ofrecer. Asimismo, una mejor conectividad y movilidad en su prestación de servicios de transporte, la facilitación y democratización de la información del destino, deberán generar prioritariamente procesos de inclusión que acerquen y comprometan al residente.

Los Destinos Turísticos Inteligentes se enmarcan dentro de un concepto de urbes inteligentes en los cuales el paradigma tecnológico atraviesa e impregna los modos o prácticas cotidianas y operativas de dicha metrópoli. De manera transversal, las TIC-piedras angulares de la innovación-, posibilitan la gestión inteligente de servicios, infraestructura, recursos y patrimonio en el espacio turístico ciudadano. Uno de los cambios fundamentales en los procesos de cogestión, administración y organización de estos espacios urbanos, radica justamente en la toma de decisiones con base al análisis de datos y su intermodalidad operativa con una matriz de red colaborativa y participativa.

En palabras de Vicini et al (2012), la apropiación tecnológica bien entendida, buscará crear sinergias con los actores y componentes sociales de la ciudad con el objetivo de mejorar y priorizar la calidad de vida de sus ciudadanos y, al mismo tiempo, mejorar la eficiencia en la prestación de bienes y servicios urbanos, generar mecanismos más dinámicos y expeditivos para resolver las dificultades cotidianas, optimizar el uso de la energía y habilitar redes útiles de movilidad y accesibilidad para sus habitantes (Vicini et al. 2012).

En esta misma línea de análisis, para Segittur (2015), la concepción de Destino Turístico Inteligente se construye a partir de considerar criterios holísticos y sistémicos, promoviendo un desarrollo sostenible y gestionando con eficiencia recursos y equipamientos turísticos. Luego, la accesibilidad universal, eje vertebrador e inclusivo de una propuesta inteligente y urbana se trasladará a una práctica de facilitación, interacción e integración del turista-visitante con el local-residente.

Según Schaffers et al. (2011) el concepto de ciudad inteligente se encuentra directamente vinculado al resultado de procesos innovadores que se integran como ecosistemas. De este modo, se potencia la co-creación de las comunidades para diseñar un modo de vida y saber hacer innovador que resulte en un verdadero cambio y compromiso dinámico y constante con 
Actas del Seminario Internacional Destinos Turísticos Inteligentes:

nuevos horizontes en la investigación y gestión del turismo

Universidad de Alicante, 26 y 27 de octubre de 2017

la totalidad de los actores. En este marco, se propiciará un uso de las TIC que garanticen la ecoeficiencia del destino pero que a su vez- sea capaz de relacionar y conectar destinos, servicios, personas e información (Big Data).

Siguiendo a Buhalis y Amaranggana (2013) en el planteo y conformación de algunas prácticas y aplicaciones del sector turístico consecuentes con la construcción y conformación de un destino turístico inteligente, podemos mencionar la experimentación y la recreación digital de atractivos y sitios turísticos a través de prácticas de realidad aumentada, sistemas de seguimiento de transporte con horarios e información disponible en tiempo real, sistemas de alojamiento capaces de predecir la demanda energética y ofrecer prestaciones eco-eficientes en la gestión del medio ambiente, aplicaciones multilingües para servicios de guías, tours y paquetes de viajes disponibles para los turistas, como así también la habilitación de códigos QR que permitan el acceso a mayor información sobre atractivos y puntos de interés turísticos a través de dispositivos móviles. Asimismo, en el marco del servicio de atención al cliente, se pone a disposición del turismo un sistema de gestión de quejas cuyo soporte, a través de diversos canales TIC como apps o telefonía celular, pueden dirigirse directamente hacia prestadores o funcionarios.

En este contexto, cuatro conceptos-eje centrales tales como la innovación, la tecnología, la accesibilidad universal y la sostenibilidad constituyen los requisitos estructurales para la creación de nuevos modelos de destinos turísticos inteligentes DTI, especificados en la Norma UNE 178501 / 2016, de reciente implementación. La mencionada norma, postula una mejora en la experiencia del turista durante todo el ciclo de vida (antes, durante y después) del viaje, así como el aumento de la competitividad y la mejora en el posicionamiento turístico del destino. Entre los aspectos positivos que resultan de su gestión e implementación, se destacan la creación de sinergias positivas de acuerdo con una estrategia común; la eficiencia en la gestión mediante la incorporación de la innovación y de la gestión de los datos-Big Data y Open Data-como factor clave, el uso de herramientas tecnológicas; la mejora de la conectividad de personas, empresas, servicios y destinos y el desarrollo sostenible en sus tres vertientes: ambiental, social y económica.

De igual modo, entendemos que la mejora de la accesibilidad universal del destino constituye un derecho y una apertura hacia potenciales mercados de cientos de millones de personas en todo el mundo, considerando que según indican fuentes especializadas casi un $15 \%$ de la población mundial padece algún tipo de discapacidad. 
Actas del Seminario Internacional Destinos Turísticos Inteligentes:

nuevos horizontes en la investigación y gestión del turismo

Universidad de Alicante, 26 y 27 de octubre de 2017

Un Destino Turístico Inteligente DTI podría pensarse como un laboratorio en permanente interacción living lab, (Buhalis y Amaranggana, 2013) que deberá facilitar herramientas tanto al residente como al turista para habilitar nuevas redes de interpretación de su oferta de recursos y atractivos turísticos generando conexiones emocionales entre el visitante y su destino, que a su vez propicien la interconectividad con otros destinos colindantes, locales y regionales. (Salessi, 2017)

\section{Destinos Turísticos Inteligentes: Una perspectiva desde la inclusión social}

En un contexto y perspectiva de inclusión social y participación comunitaria, los destinos turísticos inteligentes deberán acercar a sus residentes con los turistas y visitantes en su condición de ciudadanos relacionales «definiendo tanto a la ciudad como al destino, así como su reputación, a través de múltiples interacciones. Ellos hacen la ciudad; ellos conforman el destino» (Blanco, 2015).

En la concepción, planificación y proyección de un destino turístico inteligente DTI, el vínculo relacional y experiencial del turista y el residente debe entenderse como una correspondencia en constante tensión, dinámica, exigente, creativa y auténtica a la vez. Los DTI deberán plantearse nuevos usos y prácticas sostenibles y cotidianas de gestionar los flujos y movilidades turísticas de personas, tecnología, infraestructura social y equipamiento. (Salessi, 2017).

Comenzado el siglo XXI, las motivaciones, comportamiento y movilidades de esa persona que hoy denominamos turista, ha cambiado radicalmente. Definir su composición y perfil nos obliga a revisar sus prácticas globales inmersas en su búsqueda activa y genuina de nuevas experiencias y vivencias de viaje. De este modo, el destino turístico inteligente irá maximizando su capacidad de facilitar a sus turistas y visitantes, el uso inteligente de la ciudad por medio de prácticas creativas e inclusivas sostenidas por soluciones y plataformas tecnológicas colaborativas e interconectadas.

Ahora bien, creemos que la valoración conceptual de la inteligencia de un destino, no deriva únicamente del desarrollo de su matriz tecnológica sino que-tal cual señala Blanco (2015)-deberá evidenciarse y manifestarse en un «conjunto de perspectivas críticas para su futuro como, por ejemplo, una gobernanza cooperativa multinivel, o el mejor aprovechamiento del potencial del capital social y su capacidad tecnológica y digital innovadora.» 
Actas del Seminario Internacional Destinos Turísticos Inteligentes:

nuevos horizontes en la investigación y gestión del turismo

Universidad de Alicante, 26 y 27 de octubre de 2017

Creemos que un proceso de gestación y desarrollo de un DTI debe estructurarse sobre redes sociales participativas y consensuadas con la comunidad residente. Así, el buen uso de los datos abiertos y públicos y la gestión de la información se encauzarán hacia el bien común y el interés general.

Un destino turístico inteligente, hará uso de la tecnología al servicio de las personas, lo que en el sector turístico se traduce en potenciar al máximo esa relación experiencial que se genera entre turistas y residentes, un vínculo en el que la creatividad y la legitimidad del encuentro se pone por encima de cualquier otra práctica convencional de prestación de servicios turísticos.

En este sentido, y consecuentes con nuestro planteo y abordaje de la inteligencia y gestión de destinos turísticos sustentados en el eje de la inclusión social, entendemos que una política inclusiva, no puede soslayar el ya mencionado vínculo, más bien, lo debe promover y consolidar. La conjugación de plataformas tecnológicas, uso de las Tecnologías de la Información y Comunicación (TIC), y el desarrollo turístico sostenible (ambiental, socio-cultural-económico), deben configurar el nuevo paradigma de los destinos turísticos inteligentes. De este modo, los actores y agentes principales: turistas y residentes deberán retroalimentar permanentemente dicha correspondencia o relación en el sentido de compartir colaborativamente sus experiencias de viaje.

Los destinos turísticos inteligentes se nutren de datos, gobierno abierto, y participación ciudadana configurados en una matriz altamente flexible y dinámica cuya estructura se autodefine y se reconstruye por medio del cambio y la retroalimentación constante. Se promoverá el uso compartido de buenas prácticas con otros destinos turísticos, a fin de obtener áreas de mejora -superadoras- en cuanto a la prestación de productos y servicios turísticos de calidad, y la detección de problemáticas comunes entre municipios o comunidades, construyendo modelos replicables que actúen como guía de referencia en otras ciudades o destinos interesados en esta visión de proyecto colectivo.

Una propuesta territorial inclusiva en términos de DTI, se fundamentará en una tríada conceptual integrada por conceptos como participación, colaboración y co-creación, teniendo como eje rector la mejora de la calidad de vida de su población. Ahora bien, entendemos que a la hora de incorporar indicadores de inclusión, se deberían propiciar soluciones turísticas más creativas, que permitan al residente incorporarse al sistema turístico en todas sus variantes. Queremos enfatizar la idea de que los destinos turísticos 
Actas del Seminario Internacional Destinos Turísticos Inteligentes:

nuevos horizontes en la investigación y gestión del turismo

Universidad de Alicante, 26 y 27 de octubre de 2017

inteligentes deben responder tanto a turistas como a ciudadanos en términos de poder lograr mayor bienestar, e inclusión en el conjunto de la sociedad.

Una experiencia turística en el marco de la construcción y transformación hacia una comunidad inteligente de intereses convergentes, debe estructurarse/sostenerse en tres pilares o ideas fuerza transversales a la totalidad de las intervenciones sobre el territorio: la sostenibilidad en su sentido más profundo y abarcativo, el uso y apropiación responsable de la tecnologías de la información y la comunicación (TIC) al servicio de sus ciudadanos, y la participación activa de sus residentes.

Coincidimos con Blanco cuando sostiene que «una ciudad inteligente (...) es una ciudad con una visión integradora que aborda la transformación digital de nuestro tiempo de una manera democrática, coherente, equilibrada, eficiente y participativa.» (Blanco, 2015 p.4)

Un destino turístico inteligente deberá capitalizar estos flujos de información, conocimiento, datos y expertise que circulan en las redes, aprovechar su capacidad de distribución y conectividad democrática e inclusiva, fomentar una gobernanza abierta y poner al ciudadano de a pie como protagonista de su propio desarrollo. El mismo, con su amplia experiencia y conocimiento territorial, será el gestor de las problemáticas y soluciones reales de su tiempo, generará ideas creativas desde fuera de los gobiernos que agregarán valor en un escenario atravesado por el uso de las tecnologías digitales y la sostenibilidad.

Ahora bien, ¿̇cómo conjugar el urbanismo social inteligente en los DTI con prácticas concretas de integración social? Una ciudad innovadora, y un destino turístico que se precie de autoproclamarse inteligente debería promover espacios que acerquen inclusivamente a turistas con residentes, en el marco de una propuesta turística consecuente con una serie de prestaciones de productos y servicios turísticos creativos con los cuales legitimar ese encuentro: transporte y conectividad, sistemas de alojamiento, información e interpretación turística, servicio receptivo, apps de destinos, restauración, recreación, entre otros.

En el presente documento de investigación, partimos de la premisa de que la inclusión social es un elemento que necesita ser aplicado a los espacios turísticos, para que de esa forma inteligencia e inclusión se vinculen entre sí, defendiendo y trasladando a los espacios turísticos el derecho que tienen las personas de legitimar sus prácticas ante la actividad turística. Se 
Actas del Seminario Internacional Destinos Turísticos Inteligentes:

nuevos horizontes en la investigación y gestión del turismo

Universidad de Alicante, 26 y 27 de octubre de 2017

considera que una adecuada participación de la ciudadanía y un conocimiento apropiado de la situación, pueden ayudar a construir una ciudad inteligente a través del diseño universal para todas las personas y el principio de la participación en cada una de las actividades que conforman la cadena de valor turístico.

\section{Discusión}

En este contexto, y a modo de interrogante consecuente con el eje rector que guiará nuestro trabajo de investigación, nos preguntamos ¿Cuál es el grado de participación e implicación de las comunidades locales residentes en la práctica, gestión y concepción de los destinos turísticos inteligentes? ¿Qué dimensiones sociales, turísticas y participativas se ponen en juego en dicho territorio? ¿Cómo construir y registrar dichos indicadores?

En este contexto, es dable formularse el siguiente interrogante ¿será capaz un destino turístico con una plataforma tecnológica integrada y plural capaz de generar propuestas inclusivas que permitan a su comunidad residente transformar realidades deprimidas, mejorando su calidad de vida? ¿Cómo se traduce la inteligencia en pos de la inclusión social, en ciudades o urbes que si bien constituyen importantes destinos turísticos, observan entre sus indicadores grandes deudas sociales?

En pleno siglo XXI el turismo como un fenómeno económico y social, se sostiene en el desarrollo local y su contribución a la cohesión social. En este marco, los destinos turísticos inteligentes DTI deberían generar oportunidades concretas de integración e inclusión, creando legítimas fuentes de trabajo vinculadas a toda la cadena de valor y prestación de productos y servicios turísticos.

Pensar la inclusión desde un esquema de matriz de redes interconectadas, colaborativo, con una gran apropiación tecnológica, innovación constante, accesibilidad y creatividad; colocando a la actividad turística en la agenda del desarrollo sostenible y el crecimiento económico inclusivo; sin duda plantea retos y desafíos para repensar la gestión y gobernanza inteligente de los nuevos modelos de destinos turísticos.

\section{Conclusiones preliminares}

Conforme al análisis y revisión de diversos documentos y sitios oficiales, y a la amplia literatura académica que abunda al respecto, podemos decir que la 
Actas del Seminario Internacional Destinos Turísticos Inteligentes:

nuevos horizontes en la investigación y gestión del turismo

Universidad de Alicante, 26 y 27 de octubre de 2017

integración, participación y colaboración de la sociedad civil en el proceso de desarrollo de ciudades y destinos turísticos inteligentes resulta crucial para legitimar la construcción equitativa e inclusiva de dichos territorios. Una ciudad que genere espacios de gobernanza turística es, sin duda, una urbe más humana que atiende prioritariamente a sus ciudadanos, mejorando la calidad de las experiencias turísticas comunitarias y reforzando continuamente la relación turista-residente.

En palabras de Taleb Rifai, Secretario General de la OMT, en el marco de la inauguración del I Congreso de la OMT sobre Destinos Inteligentes celebrado en Murcia en febrero de 2017, el «turismo inteligente no es una tendencia, sino el futuro del desarrollo turístico» (Rifai, 2017).

La inteligencia territorial de un destino turístico y sus aplicaciones digitales consecuentes con servicios cada vez mas personalizados que agregan valor y diferenciación conservando su entorno socio-cultural y natural; se verá consagrada en la distribución equitativa de sus recursos y su capital social.

El marco del Año Internacional del Turismo Sostenible para el Desarrollo 2017 , nos plantea retos y desafíos para repensar el turismo entendido como un fenómeno que contribuya a mejorar la calidad de vida de las personas: turistas y residentes.

Creemos que un proceso de formación y concepción de una localidad, comunidad, urbe o espacio turístico inteligente no puede permitirse generar asimetrías o desigualdades en sus procesos de desarrollo, más aún, se deben favorecer instancias de co-gestión y manejo público-privado en cuanto a la planificación estratégica y participativa del sector turístico. Siempre poniendo a las personas en primer lugar.

Cada vez más, el sector turístico juega un rol preponderante en la agenda global debido a su capacidad de estimular el crecimiento económico, generar riqueza y empleo redistribuido por todo el territorio durante todas los períodos y estaciones del año, y por su apuesta a la diversificación de nuevos productos con inclusión social que aboguen por la protección y conservación de los recursos y bienes culturales y naturales.

Según los últimos datos y previsiones sobre turismo mundial presentados por la OMT, en el último barómetro 2017, el sector turismo crecerá en torno al 3\% y 4\% anual. Así, durante el año 2016, 1.235 millones de turistas se han trasladado fuera de su lugar de residencia habitual, un dato contundente que representa 46 millones más de turistas que el año anterior. Entendemos 
Actas del Seminario Internacional Destinos Turísticos Inteligentes:

nuevos horizontes en la investigación y gestión del turismo

Universidad de Alicante, 26 y 27 de octubre de 2017

que será imposible capitalizar el potencial del turismo en aspectos como la creación de empleo y el desarrollo socioeconómico sin el compromiso y avances hacia prácticas sostenibles, respetuosas con el medioambiente y sus comunidades locales.

Futuras investigaciones podrían extender nuestro análisis a diversas ciudades o comunidades a nivel regional, basándonos en una selección metodológica de recolección de datos empíricos que dé cuenta de entrevistas personales, encuestas y observaciones locales, con el objetivo de mostrar diferentes visiones, iniciativas e instancias superadoras vinculadas a la gestión inteligente de un destino turístico, a fin de replicar buenas prácticas y por qué no de considerar y advertir algunos de sus aspectos más débiles o negativos.

Los destinos inteligentes constituyen una herramienta fundamental para el desarrollo sostenible y no sólo aportan un impulso al sector turístico, sino a las sociedades en general. La apropiación y uso de las soluciones tecnológicas contribuyen de manera efectiva a que las decisiones puedan tomarse en el mismo territorio turístico sobre una base empírica, toda vez que permite priorizar diversas acciones y medidas anticipando futuros escenarios, premisa fundamental para una gestión responsable del turismo y sus consecuentes impactos. (OMT 2017)

Un destino turístico inteligente es el que redirige su economía para ser más competitivo, el que convoca a experiencias emocionales que sinergicen tecnología y buen vivir, que haga más eficiente la movilidad, infraestructuras y planta turística, que se traduzcan en buenas prácticas para mejorar la calidad de vida de sus habitantes, genere empleo digno y que enamore a un viajero experimentado.

Una genuina transformación hacia un destino turístico inteligente, deberá comprometer a sus principales actores a realizar reformas sociales y económicas profundamente comprometidas en pos de lograr,-sustentados en la tecnología- un verdadero avance integral y emancipador.

Sin duda, una comprometida participación ciudadana y un conocimiento apropiado del territorio, pueden coadyuvar a construir una ciudad inteligente a través del diseño universal para todas las personas y el principio de la participación en cada una de las actividades que conforman la cadena de valor turístico.

Finalmente, entendemos que en la construcción de un destino inteligente el formato de inclusión e integración social no debe ser soslayado. 
Actas del Seminario Internacional Destinos Turísticos Inteligentes:

nuevos horizontes en la investigación y gestión del turismo

Universidad de Alicante, 26 y 27 de octubre de 2017

Acordamos en las dimensiones o factores que permiten, desde distintos ámbitos académicos y profesionales, reforzar el concepto de destino turístico inteligente como son la tecnología, la sostenibilidad, la accesibilidad y la innovación. No obstante ello, estamos convencidos que la tecnología debe estar al servicio de las personas, asistiendo, incluyendo, compartiendo, educando, colaborando, acercando diferencias, y democratizando los espacios de encuentro con el otro. El turismo con todo su potencial dinamizador e innovador, deberá ser capaz de asimilar y apropiarse de estos cambios.

\section{Referencias bibliográficas}

Aenor: UNE 178501:2016 Sistema de gestión de los destinos turísticos inteligentes. Requisitos.

Aenor (2016). Gestionar el modelo turístico del siglo XXI. Disponible en: <http:// www.aenor.es/revista/314/destinos-turisticos-inteligentes.html>. Acesso em, 30.

Blanco, J. (2015). Libro blanco de los destinos turísticos inteligentes: estrategias y soluciones para fomentar la innovación el turismo digital.LID Editorial Empresarial, Madrid.

Buhalis, D., \& Amaranggana, A. (2013). Smart tourism destinations. In Information and communication technologies in tourism 2014 (pp. 553-564). Springer International Publishing.

Buhalis, D., \& Amaranggana, A. (2015). Smart tourism destinations enhancing tourism experience through personalization of services. In Information and Communication Technologies in Tourism 2015 (pp. 377-389). Springer International Publishing.

Domínguez, C. D., \& Talavera, A. S. (2016). Re-motivación en destinos turísticos, redistribución y poder. Revista CIDOB d'afers internacionals, (113), 107-122.

Macadar, M. A., \& Lheureux-De-Freitas, J. (2013, June). Porto Alegre: a Brazilian city searching to be smarter. In Proceedings of the 14th Annual International Conference on Digital Government Research (pp. 56-64). ACM.

Salessi, M.L. (2017) Destinos turísticos inteligentes con inclusión social: retos y desafíos de una agenda creativa.I Congreso Mundial de la OMT sobre Destinos Inteligentes. Murcia. España.

Sautu, R., Boniolo, P., Dalle, P., \& Elbert, R. (2005). Manual de metodología: construcción del marco teórico, formulación de los objetivos y elección de la metodología. CLACSO-Consejo Lationamericano de Ciencias Sociales. 
Actas del Seminario Internacional Destinos Turísticos Inteligentes:

nuevos horizontes en la investigación y gestión del turismo

Universidad de Alicante, 26 y 27 de octubre de 2017

Schaffers, H., Sällström, A., Pallot, M., Hernández-Muñoz, J. M., Santoro, R., \& Trousse, B. (2011, June). Integrating Living Labs with Future Internet experimental platforms for co-creating services within Smart Cities. In Concurrent enterprising (ICE), 2011 17th international conference on (pp. 1-11). IEEE.

SEGITTUR (2015) Informe sobre Destinos Turísticos Inteligentes: Construyendo el Futuro. Disponible en http://www.segittur.es/es/DTI/dti-detalle/Libro-Blanco-Destinos-Tursticos-Inteligentes-/\#.WJyIXfnhDIU.

SEGITTUR / INTELITUR (2015) « Guía de Apps turísticas 2015», Segunda edición, Madrid 2015.

Vicini, S., Bellini, S., \& Sanna, A. (2012). How to co-create Internet of things-enabled services for smarter cities. In The First International Conference on Smart Systems, Devices and Technologies (pp. 55-61). 